\title{
Free Field Construction of D-Branes in Rational Models of CFT and Gepner Models
}

\author{
Sergei E. PARKHOMENKO
}

Landau Institute for Theoretical Physics Chernogolovka, Russia

E-mail: spark@itp.ac.ru

Received October 30, 2007, in final form February 14, 2008; Published online February 23, 2008

Original article is available at http://www.emis.de/journals/SIGMA/2008/025/

\begin{abstract}
This is a review article of my recent papers on free field construction of D-branes in $N=2$ superconformal minimal models and Gepner models.

Key words: strings; D-branes; conformal field theory; free field construction; minimal models; Gepner models

2000 Mathematics Subject Classification: 17B65; 17B69; 17B81; 81R10; 81T30; 81T40; $81 \mathrm{~T} 60$
\end{abstract}

\section{Introduction}

The role of D-branes [1] in the description of certain nonperturbative degrees of freedom of strings is by now well established and the study of their dynamics has led to many new insights into String Theory. Much of this study was done in the large volume limit where geometric technique provides reliable information. The extrapolation into the stringy regime usually requires boundary conformal field theory (CFT) methods. In this approach D-brane configurations are given by conformally invariant boundary states or boundary conditions. However a complete microscopic description of these configurations are well understood only for the case of flat and toric backgrounds where the CFT on the world sheet is a theory of free fields.

The class of rational CFT's gives the interesting examples of curved string backgrounds where the construction of the boundary states can be given in principle. One of the important examples is $N=2$ supersymmetric minimal models which are building blocks of the Gepner models [2] of superstring compactification on toric Calabi-Yau manifolds. Thus, the problem of construction of D-branes in various rational models of CFT and in $N=2$ supersymmetric minimal models, in particular, is an important and interesting.

However the explicit construction of boundary states in these models is more complicated problem as against the case of flat or toric backgrounds. Although the structure of Hilbert space in rational models of CFT is naturally governed by the chiral algebra of symmetries the construction of the space of states is very nontrivial problem because it consists of highly degenerate representations of chiral algebra. This special feature of rational models hampers considerably the description of irreducible chiral algebra modules in terms of chiral algebra generators as the infinite number of singular vectors and submodules is generated by the action of chiral algebra on the highest weight vector. In other words, the modules freely generated by the chiral algebra are highly reducible. They contain infinite number of submodules which must be properly factored out to get the irreducible representations comprising the Hilbert space.

\footnotetext{
${ }^{\star}$ This paper is a contribution to the Proceedings of the Seventh International Conference "Symmetry in Nonlinear Mathematical Physics" (June 24-30, 2007, Kyiv, Ukraine). The full collection is available at http://www.emis.de/journals/SIGMA/symmetry2007.html
} 
The structure of singular vectors and submodules as well as the way of factorization can be described by resolutions of the irreducible modules [3, 4, 5, 6, 14, 16].

The free field representations and corresponding resolutions are of special interest in rational models of CFT. They are not only code the submodules structure but allow also to construct explicitly the fields of the model via the intertwining operators acting between the irreducible modules $[3,5,6]$. This property is very important because it provides a way to find the correlation functions in an explicit form. In other words, free field representation allows to find the solutions of the corresponding differential equations in an explicit form $[7,8,6,5]$ which is a nontrivial problem.

In the rational models of CFT with boundary the singular vectors problem appears again when we try to construct the Ishibashi and boundary states because we need to introduce an orthonormal basis for each irreducible representation. One can avoid this problem if we use the resolutions instead of the irreducible representations. Hence it is natural to apply the free field approach to the models with boundary. This problem has been solved in $[9,10]$, for the case of $S L(2)$ WZNW models and $N=2$ supersymmetric minimal models. The idea is to change the irreducible modules by the Fock modules resolutions and use these resolutions for Ishibashi and boundary states construction.

Notice that the construction is quite general and can be applied to any rational model of CFT with known free field realization. In addition, the free field construction can be extended for the case of Gepner models [11, 12], where the Recknagel-Schomerus boundary states as well as Recknagel permutation branes have been constructed in an explicit form by free fields. Because of boundary states in Gepner models, are defined by purely algebraic construction, the question of their geometric interpretation is nontrivial and interesting. I hope that free field approach might appears to be natural and efficient for the description of D-brane geometry in these models.

This is a review article of the papers [10] and [11]. Section 2 is devoted to the free field construction of boundary states in $N=2$ superconformal minimal models. We start with the preliminary material on $N=2$ minimal models in Subsection 2.1. In Subsection 2.2 we consider free field realization of the right-moving and left-moving $N=2$ Virasoro superalgebra currents and introduce the Fock modules, where the superalgebras are naturally acting. Then, we construct the Fock modules Ishibashi states satisfying A and B-type boundary conditions. In the next Subsection we use free field resolutions (so called butterfly resolutions) of irreducible $N=2$ Virasoro algebra modules to represent free field construction of Ishibashi states for corresponding irreducible modules. This is the main part of the construction. The problem here is to cancel the contributions from the huge number of redundant (non-physical) closed string states coming from the singular vectors. Similar to the bulk case, the non-physical states decoupling condition is equivalent to BRST invariance of the Ishibashi state, with respect to the sum of BRST charges of butterfly resolutions in the left and right-moving sectors. Then, free field construction of boundary states is given by Cardy prescription to the Ishibashi states. More recent paper [13] should be mentioned in this respect. In this paper the free field approach to $S L(2)$ WZNW models has also been considered but the BRST invariance condition which is of crucial importance has not been properly taken into account. In Subsection 2.4 we consider the closed string geometry of D-branes in $N=2$ minimal model using the free field realization.

Section 3 deals with the construction by free fields the D-branes in Gepner models. The construction is a straightforward generalization of the $N=2$ minimal model case. It is briefly discussed in Subsection 3.1. Then, we consider in Subsection 3.2 free field geometry of D-branes in closed and open string sectors. It is motivated by the conjecture that geometry of D-branes at string scales has natural description in terms of the free fields. 


\section{Free field construction of D-branes in $N=2$ supersymmetric minimal models}

\subsection{The symmetry algebra, Hilbert space and boundary conditions in $N=2$ supersymmetric minimal models}

The algebra of symmetries is given by holomorphic (or left-moving) and antiholomorphic (or right-moving) copies of $N=2$ Virasoro superalgebra

$$
\begin{aligned}
& T(z)=\sum_{n} L[n] z^{-n-2}, \quad J(z)=\sum_{n} J[n] z^{-n-1}, \\
& G^{+}(z)=\sum_{r} G^{+}[r] z^{-r-3 / 2}, \quad G^{-}(z)=\sum_{r} G^{-}[r] z^{-r-3 / 2}, \\
& \bar{T}(\bar{z})=\sum_{n} \bar{L}[n] \bar{z}^{-n-2}, \quad \bar{J}(\bar{z})=\sum_{n} \bar{J}[n] \bar{z}^{-n-1}, \\
& \bar{G}^{+}(\bar{z})=\sum_{r} \bar{G}^{+}[r] \bar{z}^{-r-3 / 2}, \quad \bar{G}^{-}(\bar{z})=\sum_{r} \bar{G}^{-}[r] \bar{z}^{-r-3 / 2}
\end{aligned}
$$

with the central charge

$$
c=3\left(1-\frac{2}{\mu}\right), \quad \mu=2,3, \ldots
$$

and the following commutation relations

$$
\begin{aligned}
& \left\{G^{+}[r], G^{-}[s]\right\}=\left(r^{2}-\frac{1}{4}\right) \frac{c}{6} \delta_{r+s, 0}+\frac{(r-s)}{2} J[r+s]+L[r+s], \\
& {\left[J[n], G^{ \pm}[r]\right]= \pm G^{ \pm}[n+r], \quad\left[L[n], G^{ \pm}[r]\right]=\left(\frac{n}{2}-r\right) G^{ \pm}[n+r],} \\
& {[L[n], J[m]]=-m J[n+m], \quad[J[n], J[m]]=n \frac{c}{3} \delta_{n+m, 0},} \\
& {[L[n], L[m]]=n\left(n^{2}-1\right) \frac{c}{12} \delta_{n+m, 0}+(n-m) L[n+m] .}
\end{aligned}
$$

As usual, the holomorphic and antiholomorphic fermionic currents in NS sector are expanded into half-integer modes and hence $r, s$ are half-integer numbers in (2.1), (2.2). While they are expanded into integer modes in $R$ sector, where $r, s$ are integer numbers.

Hilbert space of the model in NS-NS sector is a direct sum of tensor products of irreducible $N=2$ Virasoro superalgebra representations

$$
\Omega=\oplus_{h=0}^{\mu-2} \oplus_{j=h}^{-h} M_{h, j} \otimes \bar{M}_{h, j} .
$$

The irreducible modules $M_{h, j}$ are generated by the $N=2$ Virasoro superalgebra generators from the highest vectors $|h, j\rangle$ determined by the following annihilation conditions

$$
\begin{aligned}
& L[n]|h, j\rangle=0, \quad J[n]|h, j\rangle=0, \quad n>0, \\
& G^{ \pm}[r]|h, j\rangle=0, \quad r>-\frac{1}{2}, \quad L[0]|h, j\rangle=\frac{h(h+2)-j^{2}}{4 \mu}, \quad J[0]|h, j\rangle=\frac{j}{\mu}|h, j\rangle .
\end{aligned}
$$

When $j=h(j=-h)$ we have a so called chiral (anti-chiral) module generated from the chiral (anti-chiral) highest vector $|h, h\rangle(|h,-h\rangle)$ satisfying additional relation $G^{+}[-1 / 2]|h, h\rangle=0$ $\left(G^{-}[-1 / 2]|h,-h\rangle=0\right)$.

Notice that module $M_{h, j}$ is not freely generated from the highest vector. One can show [14] that there is an infinite number of submodules in the module freely generated from the highest vector $|h, j\rangle$. For example the vector which is called a singular vector

$$
\left|E^{+}\right\rangle=G^{+}\left[-\frac{1}{2}-m\right] \cdots G^{+}\left[-\frac{3}{2}\right]|h, h\rangle .
$$


satisfies the conditions similar to (2.3) and hence generates a submodule. Therefore, one has to factor out this one as well as the other submodules to get the irreducible representation $M_{h, h}$ from freely generated module. This problem is not trivial because of the number of submodules is infinite and they are intersect to each other $[14,16]$.

The $N=2$ Virasoro algebra has the following set of automorphisms which is known as spectral flow [15]

$$
\begin{aligned}
& G^{ \pm}[r] \rightarrow U^{-t} G^{ \pm}[r] U^{t}=G^{ \pm}[r \pm t], \\
& L[n] \rightarrow U^{-t} L[n] U^{t}=L[n]+t J[n]+t^{2} \frac{c}{6} \delta_{n, 0}, \quad J[n] \rightarrow U^{-t} J[n] U^{t}=J[n]+t \frac{c}{3} \delta_{n, 0},
\end{aligned}
$$

where $t \in Z$. Allowing $t$ in (2.5) to be half-integer, we obtain the isomorphism between the Hilbert space in NS and R sectors. Moreover, one can use the observation [16] that all irreducible modules can be obtained from the chiral modules $M_{h, j=h}, h=0, \ldots, \mu-2$ by the spectral flow action $U^{-t}, t=h, h-1, \ldots, 1$. Equivalently, one can restrict the set of chiral modules by the range $h=0, \ldots,\left[\frac{\mu}{2}\right]-1$ (here, $\left[\frac{\mu}{2}\right]$ denotes the integer part of $\frac{\mu}{2}$ ) and extend the spectral flow action by $t=\mu-1, \ldots, 1$. Thus, the set of irreducible modules can be labeled also by the set $\left\{(h, t) \mid h=0, \ldots,\left[\frac{\mu}{2}\right]-1, t=\mu-1, \ldots, 0\right\}$ (when $\mu$ is even and $h=\left[\frac{\mu}{2}\right]-1$ the spectral flow orbit becomes shorter: $\left.t=\left[\frac{\mu}{2}\right]-1, \ldots, 1[14]\right)$.

There are two types of boundary states preserving $N=2$ super-Virasoro algebra usually called $B$-type

$$
\begin{aligned}
& (L[n]-\bar{L}[-n])|B\rangle\rangle=0, \quad(J[n]+\bar{J}[-n])|B\rangle\rangle=0, \\
& \left.\left.\left.\left(G^{+}[r]+\imath \eta \bar{G}^{+}[-r]\right)|B\rangle\right\rangle=0, \quad\left(G^{-}[r]+\imath \eta \bar{G}^{-}[-r]\right)|B\rangle\right\rangle=0, \quad|B\rangle\right\rangle \in \Omega
\end{aligned}
$$

and $A$-type states

$$
\begin{aligned}
& (L[n]-\bar{L}[-n])|A\rangle\rangle=0, \quad(J[n]-\bar{J}[-n])|A\rangle\rangle=0, \\
& \left.\left.\left.\left(G^{+}[r]+\imath \eta \bar{G}^{-}[-r]\right)|A\rangle\right\rangle=0, \quad\left(G^{-}[r]+\imath \eta \bar{G}^{+}[-r]\right)|A\rangle\right\rangle=0, \quad|A\rangle\right\rangle \in \Omega,
\end{aligned}
$$

where $\imath=\sqrt{-1}$ and $\eta= \pm 1$.

\subsection{Free field realization and Ishibashi states in Fock modules}

Our first problem is to find a solution of the equations (2.6), (2.7) in the product of irreducible $N=2$ Virasoro superalgebra modules. In other words we are going to construct the Ishibashi state. However, the explicit construction of Ishibashi state is quite complicated problem not only because the $N=2$ Virasoro superalgebra is noncommutative but also because of the problem of singular vectors occurs. Instead, we use free field realization of $N=2$ minimal models and construct first the Ishibashi states for Fock modules which is much easy to do.

Free field realization in the holomorphic sector is given by free bosonic fields $X(z), X^{*}(z)$ and free fermionic fields $\psi(z), \psi^{*}(z)$,

$$
\begin{aligned}
& X(z)=x+X_{0} \ln (z)+\sum_{n \neq 0} \frac{X[n]}{n} z^{-n}, \quad X^{*}(z)=x^{*}+X_{0}^{*} \ln (z)+\sum_{n \neq 0} \frac{X^{*}[n]}{n} z^{-n}, \\
& \psi(z)=\sum_{r \in 1 / 2+Z} \psi[r] z^{-\frac{1}{2}-r}, \quad \psi^{*}(z)=\sum_{r \in 1 / 2+Z} \psi^{*}[r] z^{-\frac{1}{2}-r}, \\
& {\left[X^{*}[n], X[m]\right]=n \delta_{n+m, 0}, \quad\left[X[0], x^{*}\right]=1, \quad\left[X^{*}[0], x\right]=1, \quad\left[\psi^{*}[r], \psi[s]\right]_{+}=\delta_{r+s, 0} .}
\end{aligned}
$$

The currents of $N=2$ super-Virasoro algebra are given by

$$
G^{+}(z)=\psi^{*}(z) \partial X(z)-\frac{1}{\mu} \partial \psi^{*}(z), \quad G^{-}(z)=\psi(z) \partial X^{*}(z)-\partial \psi(z),
$$




$$
\begin{aligned}
& J(z)=\psi^{*}(z) \psi(z)+\frac{1}{\mu} \partial X^{*}(z)-\partial X(z), \\
& T(z)=\partial X(z) \partial X^{*}(z)+\frac{1}{2}\left(\partial^{2} X(z)+\frac{1}{\mu} \partial^{2} X^{*}(z)\right)+\frac{1}{2}\left(\partial \psi^{*}(z) \psi(z)-\psi^{*}(z) \partial \psi(z)\right) .
\end{aligned}
$$

It is clear that $N=2$ Virasoro superalgebra is acting in the Fock module $F_{p, p^{*}}$ which is generated by creation operators of the fields $X(z), X^{*}(z), \psi(z), \psi^{*}(z)$ from the vacuum vector $\left|p, p^{*}\right\rangle \in F_{p, p^{*}}$. It is determined by

$$
\begin{aligned}
& \psi[r]\left|p, p^{*}\right\rangle=\psi^{*}[r]\left|p, p^{*}\right\rangle=0, \quad r \geq \frac{1}{2}, \quad X[n]\left|p, p^{*}\right\rangle=X^{*}[n]\left|p, p^{*}\right\rangle=0, \quad n \geq 1, \\
& X_{0}\left|p, p^{*}\right\rangle=p\left|p, p^{*}\right\rangle, \quad X_{0}^{*}\left|p, p^{*}\right\rangle=p^{*}\left|p, p^{*}\right\rangle .
\end{aligned}
$$

The vector $\left|p, p^{*}\right\rangle$ satisfies the conditions (2.3) where

$$
h=p^{*}+\mu p, \quad j=p^{*}-\mu p .
$$

Analogously, the Fock module $\bar{F}_{\bar{p}, \bar{p}^{*}}$, which is generated from vacuum $\left|\bar{p}, \bar{p}^{*}\right\rangle$ by the creation operators of the antiholomorphic fields $\bar{X}, \bar{X}^{*}, \bar{\psi}, \bar{\psi}^{*}$, is the representation for $N=2$ Virasoro algebra in the right-moving sector.

In the tensor product of Fock modules we are going to construct Ishibashi state

$$
\left.\left|p, p^{*}, \bar{p}, \bar{p}^{*}\right\rangle\right\rangle \in F_{p, p^{*}} \otimes \bar{F}_{\bar{p}, \bar{p}^{*}}
$$

which fulfills the relations (2.6) for example. One can show that (2.6) follows from

$$
\begin{aligned}
& \left.\left.\left(\psi^{*}[r]-\imath \eta \bar{\psi}^{*}[-r]\right)\left|p, p^{*}, \bar{p}, \bar{p}^{*}, B\right\rangle\right\rangle=0, \quad(\psi[r]-\imath \eta \bar{\psi}[-r])\left|p, p^{*}, \bar{p}, \bar{p}^{*}, B\right\rangle\right\rangle=0, \\
& \left.\left(X[n]+\bar{X}[-n]+\frac{\delta_{n, 0}}{\mu}\right)\left|p, p^{*}, \bar{p}, \bar{p}^{*}, B\right\rangle\right\rangle=0, \\
& \left.\left(X^{*}[n]+\bar{X}^{*}[-n]+\delta_{n, 0}\right)\left|p, p^{*}, \bar{p}, \bar{p}^{*}, B\right\rangle\right\rangle=0, \\
& \bar{p}=-p-\frac{1}{\mu}, \quad \bar{p}^{*}=-p^{*}-1 .
\end{aligned}
$$

The solution is given by a standard expression

$$
\begin{aligned}
\left.\left|p, p^{*}, B\right\rangle\right\rangle= & \prod_{n=1} \exp \left(-\frac{1}{n} X^{*}[-n] \bar{X}[-n]\right) \exp \left(-\frac{1}{n} X[-n] \bar{X}^{*}[-n]\right) \\
& \left.\times \prod_{r=1 / 2} \exp \left(\imath \eta \psi^{*}[-r] \bar{\psi}[-r]\right) \exp \left(\imath \eta \psi[-r] \bar{\psi}^{*}[-r]\right)\right)\left|p, p^{*},-p-\frac{1}{\mu},-p^{*}-1\right\rangle
\end{aligned}
$$

The $A$-type Ishibashi states can be found analogously. The relations (2.7) follow from

$$
\begin{aligned}
& \left.\left.\left(\psi^{*}[r]-\imath \eta \mu \bar{\psi}[-r]\right)\left|p, p^{*}, \bar{p}, \bar{p}^{*}, \eta, A\right\rangle\right\rangle=0, \quad\left(\psi[r]-\imath \frac{\eta}{\mu} \bar{\psi}^{*}[-r]\right)\left|p, p^{*}, \bar{p}, \bar{p}^{*}, \eta, A\right\rangle\right\rangle=0, \\
& \left.\left(\mu X[n]+\bar{X}^{*}[-n]+\delta_{n, 0}\right)\left|p, p^{*}, \bar{p}, \bar{p}^{*}, \eta, A\right\rangle\right\rangle=0, \\
& \left.\left(X^{*}[n]+\mu \bar{X}[-n]+\delta_{n, 0}\right)\left|p, p^{*}, \bar{p}, \bar{p}^{*}, \eta, A\right\rangle\right\rangle=0 \\
& \bar{p}=-\frac{1+p^{*}}{\mu}, \quad \bar{p}^{*}=-\mu-1 .
\end{aligned}
$$

Hence the $A$-type Fock space Ishibashi state (in NS sector) is given by

$$
\begin{aligned}
\left.\left|p, p^{*}, \eta, A\right\rangle\right\rangle= & \prod_{n=1} \exp \left(-\frac{1}{n}\left(\mu X[-n] \bar{X}[-n]+\frac{1}{\mu} X^{*}[-n] \bar{X}^{*}[-n]\right)\right) \\
& \times \prod_{r=1 / 2} \exp \left(\imath \eta\left(\frac{1}{\mu} \psi^{*}[-r] \bar{\psi}^{*}[-r]+\mu \psi[-r] \bar{\psi}[-r]\right)\right)\left|p, p^{*},-\frac{1+p^{*}}{\mu},-\mu p-1\right\rangle .
\end{aligned}
$$




\subsection{Ishibashi states in irreducible $N=2$ Virasoro superalgebra modules and boundary states in $N=2$ minimal model}

How to use the solution (2.9) to build the Ishibashi state for irreducible $N=2$ Virasoro superalgebra modules?

The Fock module $F_{p, p^{*}}$ is highly reducible representation. It contains infinite number of singular (and cosingular) vectors generating $N=2$ Virasoro submodules similar to the example (2.4). Therefore, the state (2.9) contains contributions from huge number of redundant states coming from singular vectors.

In other words, the overlap of this state with an arbitrary closed string state which does not belong to the Hilbert space of the $N=2$ minimal model is not zero in general

$$
\left\langle\left\langle p, p^{*}, \eta, B \mid v \otimes \bar{v}\right\rangle \neq 0, \quad \mid v \otimes \bar{v}\right\rangle \in F_{p, p^{*}} \otimes \bar{F}_{\bar{p}, \bar{p}^{*}}, \quad|v \otimes \bar{v}\rangle \notin \Omega .
$$

If we want to build the Ishibashi state for irreducible representation it is necessary to cancel contributions from submodules.

Hence we have to investigate the structure of submodules in the Fock modules and extract the irreducible representations. For the module $F_{0, h}$ it is given by the following infinite complex [16] (which is known due to Feigin and Semikhatov as butterfly resolution)
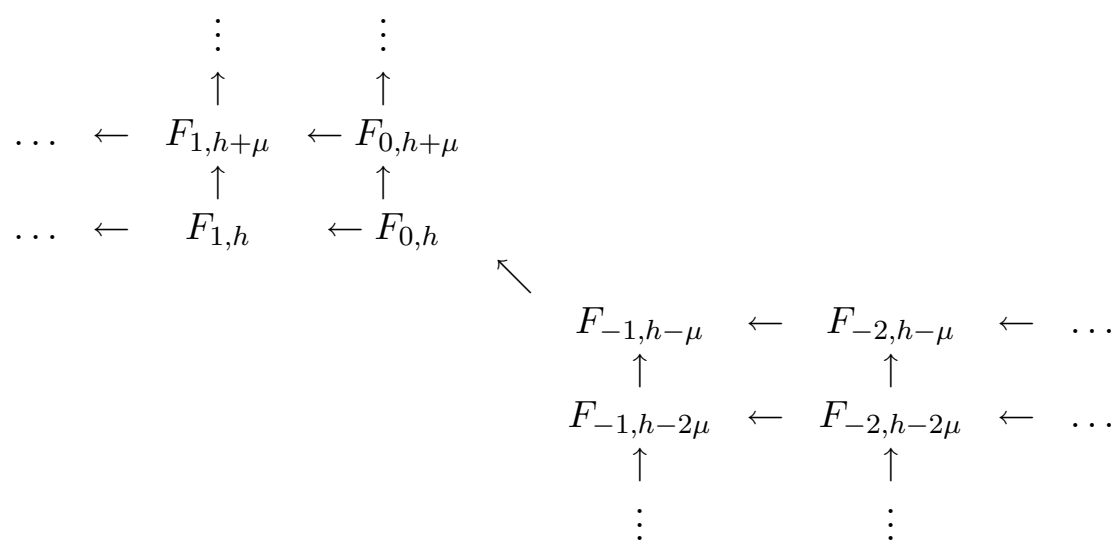

The horizontal arrows in this diagram are given by the action of screening charge

$$
Q^{+}=\oint d z \psi^{*} \exp \left(X^{*}\right)
$$

and vertical arrows are given by the action of screening charge

$$
Q^{-}=\oint d z \psi \exp (\mu X)
$$

The diagonal arrow at the middle of butterfly resolution is given by the action of $Q^{+} Q^{-}$. The ghost number $g$ of the complex is increasing along the diagonal from the right to the left and $F_{0, h}$ module is ghost number zero subspace.

The crucial property of the $Q^{ \pm}$operators is that they commute to the Virasoro algebra generators and they are nilpotent

$$
\left[L[n], Q^{ \pm}\right]=\left[J[n], Q^{ \pm}\right]=\cdots=0, \quad\left(Q^{ \pm}\right)^{2}=0, Q^{+} Q^{-}+Q^{-} Q^{+}=0 .
$$

Hence the $Q^{ \pm}$-images of the Fock modules are the $N=2$ Virasoro superalgebra submodules. In particular, $Q^{ \pm}$-images of the vacuum vectors $\left|p, p^{*}\right\rangle$ are the singular vectors like (2.4). 
Theorem 1 ([16]). Complex (2.11) is exact except at the $F_{0, h}$ module, where the cohomology is given by the chiral module $M_{h, j=h}$.

What about the singular vector structure for the module $\bar{F}_{-\frac{1}{\mu},-1-h}$ coming from the rightmoving sector of the state (2.9)? It is given by dual butterfly resolution [10].

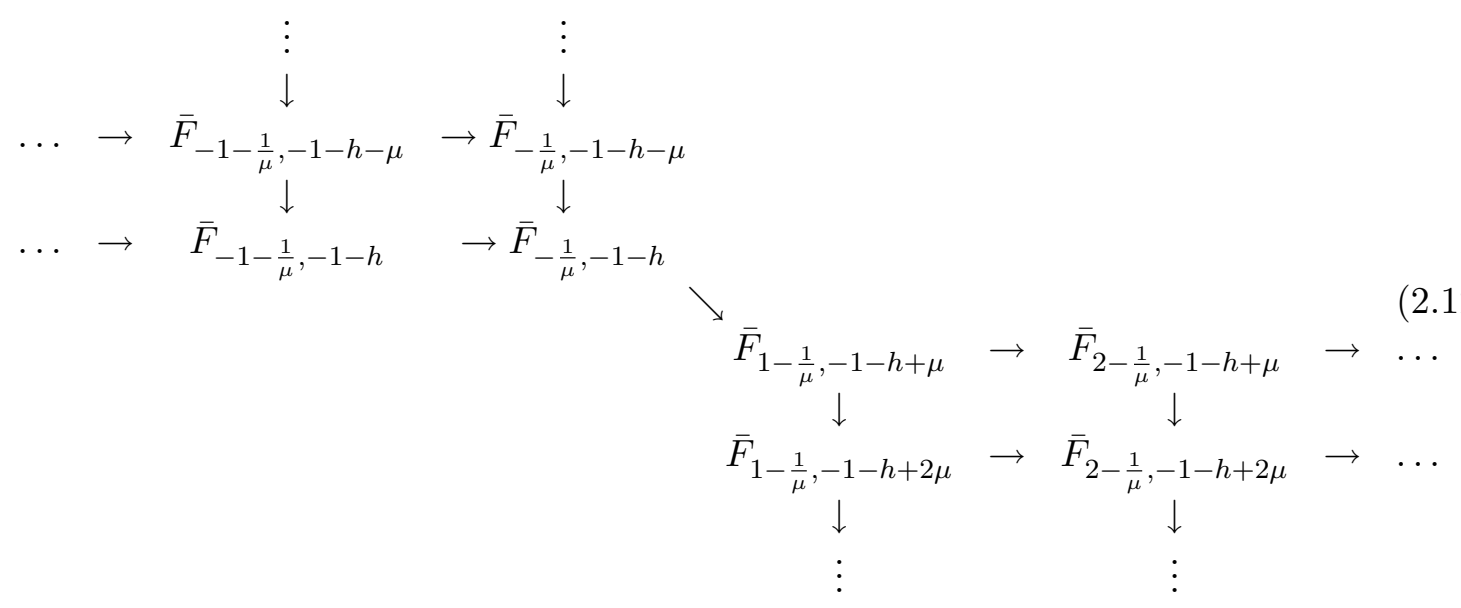

The arrows on this diagram are given by the same operators as on the diagram (2.11).

Theorem 2 ([10]). Complex (2.12) is exact except at the $\bar{F}_{-\frac{1}{\mu},-1-h}$ module, where the cohomology is given by the anti-chiral irreducible module $M_{h, j=-h}$.

To make the cancelation of redundant states from submodules we add (with appropriate coefficients) the Fock Ishibashi states (2.9) from the tensor products of modules forming the butterfly complex (2.11) and its dual (2.12). Thus, the free field construction of the Ishibashi state for the irreducible representation

$$
\left.\left|M_{h, j=h}, \eta, B\right\rangle\right\rangle \in M_{h, j=h} \otimes \bar{M}_{h, j=-h}
$$

is given by the superposition

$$
\left.\left.\left.\left|M_{h, j=h}, \eta, B\right\rangle\right\rangle=\sum_{n, m \geq 0} c_{n, m}|n, m \mu+h, \eta, B\rangle\right\rangle+\sum_{n, m>0} c_{-n,-m}|-n,-m \mu+h, \eta, B\rangle\right\rangle
$$

where the coefficients $c_{n, m}$ are fixed by the BRST invariance condition which is equivalent to the redundant states cancelation.

BRST invariance condition can be formulated as follows. First of all, we form a tensor product of the butterfly complexes (2.11) and (2.12)

$$
\cdots \rightarrow C_{h, h}^{-2} \rightarrow C_{h, h}^{-1} \rightarrow C_{h, h}^{0} \rightarrow C_{h, h}^{+1} \rightarrow \cdots,
$$

which is graded by the sum of the ghost numbers $g+\bar{g}$. The differential $D$ of the complex is defined by the differentials $d$ and $\bar{d}$ of the complexes (2.11) and (2.12)

$$
D\left|v_{g} \otimes \bar{v}_{g^{\prime}}\right\rangle=\left|d v_{g} \otimes \bar{v}_{g^{\prime}}\right\rangle+(-1)^{g}\left|v_{g} \otimes \bar{d}_{g^{\prime}}\right\rangle,
$$

where $\left|v_{g}\right\rangle$ is an arbitrary vector from the complex (2.11) with the ghost number $g$, while $\left|\bar{v}_{g^{\prime}}\right\rangle$ is an arbitrary ghost number $g^{\prime}$ vector from the complex (2.12).

It follows from the theorems above that the cohomology of the complex (2.14) is centered at zero grading

$$
\mathbf{H}^{0}=M_{h, h=j} \otimes \bar{M}_{h, j=-h}, \quad \mathbf{H}^{g}=0, \quad g \neq 0 .
$$


The Ishibashi state we are looking for can be considered as a linear functional on the Hilbert space of $N=2$ superconformal minimal model. Therefore, the BRST invariance condition can be written as follows:

$$
\left.D^{*}\left|M_{h, j=h}, \eta, B\right\rangle\right\rangle=0 \quad \Leftrightarrow \quad\left\langle\left\langle M_{h, h}, \eta, B|D| v_{g} \otimes \bar{v}_{i-g}\right\rangle=0, \quad \mid v_{g} \otimes \bar{v}_{i-g}\right\rangle \in C_{h, h}^{i} .
$$

From this equation we find [10] that coefficients of the superposition $c_{n, m}$ are proportional to $c_{0,0}$ according to the ghost numbers of the double complex (2.14)

$$
c_{n, m}=\exp \left(\imath \eta \frac{\pi}{2}(n+m)^{2}\right) c_{0,0} .
$$

It gives explicit free field construction of Ishibashi state $\left.\left|M_{h, j=h}\right\rangle\right\rangle$.

The free field construction of the Ishibashi states for the modules $M_{h, j}, j \neq h$ is given by the spectral flow action $U^{t}$, where $t=0, \ldots, h$

$$
\left.\left.\left|M_{h, t}, \eta, B\right\rangle\right\rangle=U^{t}(\bar{U})^{-t}\left|M_{h}, \eta, B\right\rangle\right\rangle \text {. }
$$

Analogously, the free field representation of $A$-type irreducible module Ishibashi state is given by the following superposition of Fock Ishibashi states

$$
\begin{aligned}
& \left.\left.\left|M_{h, t}, \eta, A\right\rangle\right\rangle=U^{t}(\bar{U})^{t}\left|M_{h}, \eta, A\right\rangle\right\rangle, \\
& \left.\left.\left.\left|M_{h}, \eta, A\right\rangle\right\rangle=\sum_{n, m \geq 0} c_{n, m}|n, m \mu+h, \eta, A\rangle\right\rangle+\sum_{n, m>0} c_{-n,-m}|-n,-m \mu+h, \eta, A\rangle\right\rangle,
\end{aligned}
$$

where another normalization of the screening charges $\bar{Q}^{ \pm}$has been used to cancel $\mu$ and $\frac{1}{\mu}$ factors for $c_{n, m}$ coefficients.

Now the free field construction of $A$ and $B$-type boundary states (in NS sector) in $N=2$ minimal models is given by Cardy prescription [17]

$$
\begin{aligned}
\left.\left|D_{h, t}, \eta, A\right\rangle\right\rangle & \left.=\sum_{h^{\prime}, t^{\prime}} D_{(h, t),\left(h^{\prime}, t^{\prime}\right)}\left|M_{h^{\prime}, t^{\prime}}, \eta, A\right\rangle\right\rangle, \\
\left.\left|D_{h, t}, \eta, B\right\rangle\right\rangle & \left.=\sum_{h^{\prime}=\text { even }} D_{(h, t),\left(h^{\prime}, t^{\prime}=h^{\prime} / 2\right)}\left|M_{h^{\prime}, t=h^{\prime} / 2}, \eta, B\right\rangle\right\rangle,
\end{aligned}
$$

where $D_{(h, t),\left(h^{\prime}, t^{\prime}\right)}$ are Cardy coefficients determined by $N=2$ minimal model modular matrix

$$
S_{(h, t),\left(h^{\prime}, t^{\prime}\right)}=\frac{1}{\sqrt{2} \mu} \sin \left(\frac{\pi(h+1)\left(h^{\prime}+1\right)}{\mu}\right) \exp \left(\imath \pi \frac{(h-2 t)\left(h^{\prime}-2 t^{\prime}\right)}{\mu}\right) .
$$

Thus, the coefficients $c_{0,0}$ in the superpositions (2.18) should be identified with the Cardy's coefficients.

In conclusion of this section we notice that the free field representations of boundary states are determined modulo BRST-exact states satisfying $A$ or $B$-type boundary conditions. We interpret this ambiguity in the free field representation as a result of adding brane-antibrane pairs annihilating under the tachyon condensation process [18]. Strictly speaking, the BRST-exact states ambiguity is not the usual brane-antbrane pair ambiguity and has to be considered in a generalized sense, because BRST-exact states contain also the states with negative charges in NS sector. In particular, there are the states in NS sector oppositely charged with respect to graviton. Similar objects has recently been discussed in [20]. In this context the free field representation (2.19) of boundary states can be considered as the superposition of branes flowing under the (generalized) tachyon condensation to nontrivial boundary states in minimal model. It is also important to note that spectral flow automorphisms (2.5) give different free field representations of boundary states because the corresponding butterfly resolutions are not invariant with respect to these automorphisms. However, their cohomology is invariant. Hence, these different representations have to be identified and the free field boundary state construction should be considered in the sense of derived category [19]. 


\subsection{Free field geometry of D-branes in $N=2$ minimal models}

Introducing the new set of bosonic and fermionic oscillators

$$
\begin{array}{rlrl}
v[n] & =\frac{\imath}{\sqrt{2 \mu}}\left(X^{*}[n]-\mu X[n]\right), & & u[n]=\frac{1}{\sqrt{2 \mu}}\left(X^{*}[n]+\mu X[n]\right), \\
\bar{v}[n] & =\frac{\imath}{\sqrt{2 \mu}}\left(\bar{X}^{*}[n]-\mu \bar{X}[n]\right), & & \bar{u}[n]=-\frac{1}{\sqrt{2 \mu}}\left(\bar{X}^{*}[n]+\mu \bar{X}[n]\right), \\
\sigma[r] & =\frac{1}{\sqrt{2 \mu}}\left(\psi^{*}[r]+\mu \psi[r]\right), & \gamma[r]=\frac{\imath}{\sqrt{2 \mu}}\left(\psi^{*}[r]-\mu \psi[r]\right), \\
\bar{\sigma}[r]=-\frac{1}{\sqrt{2 \mu}}\left(\bar{\psi}^{*}[r]+\mu \bar{\psi}[r]\right), & \bar{\gamma}[r]=\frac{\imath}{\sqrt{2 \mu}}\left(\bar{\psi}^{*}[r]-\mu \bar{\psi}[r]\right) .
\end{array}
$$

one can rewrite the $A$ and $B$-type boundary conditions (2.10), (2.8) as

$$
\begin{aligned}
& \left.\left.\left(u[n]-\bar{u}[-n]+\sqrt{\frac{2}{\mu}} \delta_{n, 0}\right)\left|p, p^{*}, \bar{p}, \bar{p}^{*}, \eta, A\right\rangle\right\rangle=0, \quad(v[n]-\bar{v}[-n])\left|p, p^{*}, \bar{p}, \bar{p}^{*}, \eta, A\right\rangle\right\rangle=0, \\
& \left.\left.(\gamma[r]+\imath \eta \bar{\gamma}[-r])\left|p, p^{*}, \bar{p}, \bar{p}^{*}, \eta, A\right\rangle\right\rangle=0, \quad(\sigma[r]-\imath \eta \bar{\sigma}[-r])\left|p, p^{*}, \bar{p}, \bar{p}^{*}, \eta, A\right\rangle\right\rangle=0, \\
& \left.\left.\left(u[n]-\bar{u}[-n]+\sqrt{\frac{2}{\mu}} \delta_{n, 0}\right)\left|p, p^{*}, \bar{p}, \bar{p}^{*}, \eta, B\right\rangle\right\rangle=0, \quad(v[n]+\bar{v}[-n])\left|p, p^{*}, \bar{p}, \bar{p}^{*}, \eta, B\right\rangle\right\rangle=0, \\
& \left.\left.(\gamma[r]+\imath \eta \bar{\gamma}[-r])\left|p, p^{*}, \bar{p}, \bar{p}^{*}, \eta, B\right\rangle\right\rangle=0, \quad(\sigma[r]-\imath \eta \bar{\sigma}[-r])\left|p, p^{*}, \bar{p}, \bar{p}^{*}, \eta, B\right\rangle\right\rangle=0 .
\end{aligned}
$$

Thus, $A$-type states correspond to Dirichlet boundary condition along the coordinates $u, v$ and $B$-type states correspond to Dirichlet boundary condition along the coordinate $u$ and to Neumann boundary condition along the coordinate $v$. Notice also that we can view the coordinates $\exp (u), v$ as polar coordinates on the complex plane because of the currents $J(z), T(z)$ take the form

$$
J(z)=\psi^{*}(z) \psi(z)-\imath \sqrt{\frac{2}{\mu}} \partial v(z), \quad T(z)=\frac{1}{2}\left(\partial u^{2}+\partial v^{2}\right)-\frac{1}{\sqrt{2 \mu}} \partial^{2} u .
$$

Hence, in these coordinates $A$-type states are the points on the complex plane and $B$-type states correspond to 1-dimensional circles around the origin. It is clear however, that we are free to change arbitrary the sings in front of the right-moving coordinates in (2.20) changing thereby the boundary conditions. The definition (2.20) stems from the consistency with the expressions (2.13), (2.17), (2.18) and our wish to have a geometry close to geometry of LG model. Indeed, due to the summation over $n+m$ in (2.13) and (2.18) it is natural to view the boundary conditions as Dirichlet along the coordinate $u$, which is noncompact direction, so that the winding states are not allowed in this direction by topology. The summation over the $n-m$ is natural to consider as a summation over the winding modes in $B$-type branes and summation over the momenta in $A$-type branes. In this picture, $A$-type boundary conditions correspond to D0-branes while $B$-type boundary conditions correspond to D1-branes. It should be noted once more that this geometric interpretation is quite artificial and it would be interesting to find a CFT argument to fix the ambiguity of boundary conditions interpretation.

\section{Free field construction of D-branes in Gepner models}

In Gepner models of superstring compactification the string degrees of freedom on the compact manifold are given by internal $N=2 \mathrm{CFT}$ which is direct product of $N=2$ supersymmetric minimal models factored out by GSO projection

$$
\Omega \approx M_{1} \times M_{2} \times \cdots \times M_{I} / \mathrm{GSO}, \quad c=\sum_{i=1}^{I} c_{i}=9, \quad c_{i}=3\left(1-\frac{2}{\mu_{i}}\right) .
$$

The $N=2$ Virasoro superalgebra of the model is given by the diagonal in the product of individual $N=2$ Virasoro superalgebras

$$
L[n]=\left(L_{(1)}+\cdots+L_{(I)}\right)[n], \quad J[n]=\left(J_{(1)}+\cdots+J_{(I)}\right)[n], \quad \cdots
$$


The GSO projection group in the internal sector is generated by the operator

$$
G_{\mathrm{GSO}}=\exp (2 \imath \pi(J[0]+\bar{J}[0]))
$$

and hence the diagonal $N=2$ Virasoro algebra survives the projection. Thus, we have purely algebraic construction of the Hilbert space [2] (see also [22, 23]).

It is obvious that free field representation of D-branes in Gepner models can be obtained from the free field construction of D-branes in $N=2$ minimal models if we take into account the GSO projection [11].

\subsection{Free field representation of D-branes}

In the free field language we introduce in the left-moving sector the free bosonic fields $X_{i}(z)$, $X_{i}^{*}(z)$ and free fermionic fields $\psi_{i}(z), \psi_{i}^{*}(z), i=1, \ldots, I$ as well as the lattice of momenta

$$
\begin{aligned}
& \Pi=P \oplus P^{*}, \\
& P=\mathbf{p}=\left(\frac{n_{1}}{\mu_{1}}, \ldots, \frac{n_{I}}{\mu_{I}}\right), \quad n_{i} \in Z, \quad P^{*}=\mathbf{p}^{*}=\left(m_{1}, \ldots, m_{I}\right), \quad m_{i} \in Z
\end{aligned}
$$

and the set of Fock modules associated to this lattice

$$
F_{\mathbf{p}, \mathbf{p}^{*}}, \quad\left(\mathbf{p}, \mathbf{p}^{*}\right) \in \Pi \text {. }
$$

The corresponding butterfly resolution is given by the product of butterfly resolutions of individual minimal models. Hence the differential is given by the screening charges

$$
Q_{i}^{+}=\oint d z \psi_{i}^{*} \exp \left(X_{i}^{*}\right), \quad Q_{i}^{-}=\oint d z \psi_{i} \exp \left(\mu_{i} X_{i}\right)
$$

The similar objects have to be introduced in the right-moving sector.

Thus, according to (2.18) the free field construction of $A$-type Ishibashi state in the product of minimal models is given by

$$
\left.\left.\left.\left.\left|M_{\mathbf{h}, \mathbf{t}}, \eta, A\right\rangle\right\rangle=\prod_{i} U_{i}^{-t_{i}} \bar{U}_{i}^{-t_{i}}\left|M_{\mathbf{h}}, \eta, A\right\rangle\right\rangle, \quad\left|M_{\mathbf{h}}, \eta, A\right\rangle\right\rangle=\sum_{\left(\mathbf{p}, \mathbf{p}^{*}\right) \in \Gamma_{\mathbf{h}}} c_{\mathbf{p}, \mathbf{p}^{*}}\left|\mathbf{p}, \mathbf{p}^{*}, \eta, A\right\rangle\right\rangle,
$$

where $\Gamma_{\mathbf{h}}$ is the set of butterfly resolution momenta and

$$
\begin{aligned}
& \left.\left|\mathbf{p}, \mathbf{p}^{*}, \eta, A\right\rangle\right\rangle=\prod_{n=1} \exp \left(-\frac{1}{n} \sum_{i}\left(\mu_{i} X_{i}[-n] \bar{X}_{i}[-n]+\frac{1}{\mu_{i}} X_{i}^{*}[-n] \bar{X}_{i}^{*}[-n]\right)\right) \\
& \quad \times \prod_{r=1 / 2} \exp \left(\imath \eta \sum_{i}\left(\mu_{i} \psi_{i}[-r] \bar{\psi}_{i}[-r]+\frac{1}{\mu_{i}} \psi_{i}^{*}[-r] \bar{\psi}_{i}^{*}[-r]\right)\right)\left|\mathbf{p}, \mathbf{p}^{*},-\Omega^{-1} \mathbf{p}^{*}-\mathbf{d},-\Omega \mathbf{p}-\mathbf{d}^{*}\right\rangle,
\end{aligned}
$$

where we have introduced the matrix $\Omega_{i j}=\mu_{i} \delta_{i j}$. Now the construction of $A$-type boundary states is straightforward, we use the Cardy prescription and the GSO projection

$$
\left.|\boldsymbol{\Lambda}, \lambda, \eta, A\rangle\rangle=\frac{\omega}{\kappa^{2}} \sum_{(\mathbf{h}, \mathbf{t}) \in \Delta} W_{\boldsymbol{\Lambda}, \lambda}^{\mathbf{h}, \mathbf{t}} \sum_{m, n=0}^{\kappa-1} \exp \left(\imath 2 \pi n J_{0}\right) U^{m \mathbf{v}} \bar{U}^{m \mathbf{v}}\left|M_{\mathbf{h}, \mathbf{t}}, \eta, A\right\rangle\right\rangle,
$$

where $W_{\boldsymbol{\Lambda}, \lambda}^{\mathbf{h}, \mathbf{t}}$ are Cardy's coefficients

$$
W_{\boldsymbol{\Lambda}, \lambda}^{\mathbf{h}, \mathbf{t}}=R_{\Lambda}^{\mathbf{h}} \exp \left(\imath \pi \sum_{i=1} \frac{\left(\Lambda_{i}-2 \lambda_{i}\right)\left(h_{i}-2 t_{i}\right)}{\mu_{i}}\right), \quad R_{\boldsymbol{\Lambda}}^{\mathbf{h}}=\prod_{i=1}^{r} R_{\Lambda_{i}}^{h_{i}},
$$




$$
R_{\Lambda_{i}}^{h_{i}}=\frac{S_{\Lambda_{i}, h_{i}}}{\sqrt{S_{0, h_{i}}}}, \quad S_{\Lambda_{i}, h_{i}}=\frac{\sqrt{2}}{\mu_{i}} \sin \left(\frac{\pi\left(\Lambda_{i}+1\right)\left(h_{i}+1\right)}{\mu_{i}}\right)
$$

$\Delta$ is the set of irreducible representations from the product of minimal models and $\omega$ is the normalization constant. The summation over $n$ makes projection on the set of integer $J[0]$ charges providing thereby GSO projection in the internal sector of the superstring [23, 21], while the summation over $m$ introduce spectral flow twisted sectors. The boundary states are labelled by pair of vectors $(\boldsymbol{\Lambda}, \lambda)=\left(\Lambda_{1}, \ldots, \Lambda_{I}, \lambda_{1}, \ldots, \lambda_{I}\right) \in \Delta$.

The $B$-type boundary states are given by the similar expression [11]. Thus, we obtain the construction of Recknagel-Schomerus boundary states [21] in an explicit form. The free field representation can be generalized also to the case of permutation branes of Recknagel [24]. It was given in [12].

\subsection{Free field geometry of D-branes in Gepner models}

The geometry of D-branes analysis in the closed string sector is given analogously to the minimal models. Similar to $(2.20)$ we introduce in the left-moving sector the bosonic fields $v_{i}(z), u_{i}(z)$, the fermionic fields $\sigma_{i}(z), \gamma_{i}(z)$ and do the same in the right-moving sector, introducing $\bar{v}_{i}(\bar{z})$, $\bar{u}_{i}(\bar{z}), \bar{\sigma}_{i}(\bar{z}), \bar{\gamma}_{i}(\bar{z}), i=1, \ldots, I$. It is clear that in this picture $A$-type boundary conditions in the Gepner model are Dirichlet-ones for all coordinates, while $B$-type boundary conditions are Dirichlet conditions for $u_{i}$ coordinates and Neumann-ones for $v_{i}$ coordinates. Hence, before the GSO projection the $A$-type boundary state corresponds to D0-brane in the flat complex space $\mathbb{C}^{I}$ and $B$-type boundary state describes a Lagrangian $I$-dimensional torus in $\mathbb{C}^{I}$, where $\exp \left(u_{i}\right)$, and $v_{i}$ are the polar coordinates. Due to (2.20), (2.21) and (3.1) it is easy to see how the GSO group is acting in $\mathbb{C}^{I}$, it makes the orbifold $\mathbb{C}^{I} /$ GSO. Hence the $A$ and $B$-type boundary states in Gepner models are the D0 and DI-branes on the orbifold. The question which seems to me important is to understand what is geometric meaning of Cardy's coefficients and how they code the moduli space of D-branes in Gepner models.

There is another aspect of the free field geometry of D-branes in Gepner models. It appears in the open string sector of D-branes [11, 12]. In this case the space of states of the open strings between the pair of D-branes can be described by one of the sectors, the left-moving one for example. It is easy to calculate the transition amplitude between the pair of boundary states. The result comes from the BRST construction of Ishibashi states (2.15), (2.16) as well as representation of the irreducible modules characters in the $N=2$ minimal model by an alternating sum of the Fock modules characters from the butterfly resolution [16]:

$$
\begin{aligned}
& Z_{\left(\boldsymbol{\Lambda}_{1}, \lambda_{1}\right)\left(\boldsymbol{\Lambda}_{2}, \lambda_{2}\right)}^{A}(q) \equiv\left\langle\left\langle\boldsymbol{\Lambda}_{1}, \lambda_{1}, \eta, A\left|(-1)^{g} \tilde{q}^{L_{0}-\frac{c}{24}}\right| \boldsymbol{\Lambda}_{2}, \lambda_{2}, \eta, A\right\rangle\right\rangle \\
& \quad=\omega^{2} \sum_{\mathbf{h}, \mathbf{t}} \prod_{i}\left(N_{\Lambda_{1, i}, \Lambda_{2, i}}^{h_{i}} \delta^{\left(2 \mu_{i}\right)}\left(\Lambda_{2, i}-2 \lambda_{2, i}-\Lambda_{1, i}+2 \lambda_{1, i}+h_{i}-2 t_{i}\right)\right. \\
& \left.\quad+N_{\Lambda_{1, i}, \Lambda_{2, i}}^{\mu_{i}-h_{i}-2} \delta^{\left(2 \mu_{i}\right)}\left(\Lambda_{2, i}-2 \lambda_{2, i}-\Lambda_{1, i}+2 \lambda_{1, i}+h_{i}-2 t_{i}-\mu_{i}\right)\right) \operatorname{ch}_{\mathbf{h}, \mathbf{t}}(q),
\end{aligned}
$$

where $(-1)^{g}$ is the ghost number insertion according to BRST condition $(2.15), q=\exp (2 \imath \pi \tau)$, $\tilde{q}=\exp \left(-2 \imath \frac{\pi}{\tau}\right), N_{\Lambda_{1, i}, \Lambda_{2, i}}^{h_{i}}$ are Verlinde algebra coefficients for the individual minimal model, and

$$
\begin{aligned}
\operatorname{ch}_{\mathbf{h}, \mathbf{t}}(q, u) & =\frac{1}{\kappa^{2}} \sum_{n, m=0}^{\kappa-1} \operatorname{Tr}_{M_{\mathbf{h}, \mathbf{t}}}\left(U^{n \mathbf{v}} q^{\left(L_{0}-\frac{c}{24}\right)} u^{J_{0}} \exp \left(2 \imath \pi m J_{0}\right) U^{-n \mathbf{v}}\right) \\
& =\frac{1}{\kappa^{2}} \sum_{n, m=0}^{\kappa-1} \chi_{\mathbf{h}, \mathbf{t}+n \mathbf{v}}(\tau, v+m)
\end{aligned}
$$


where $M_{\mathbf{h}, \mathbf{t}}=\prod_{i} M_{h_{i}, t_{i}}$ is the product of irreducible modules, $U^{n \mathbf{v}}=\prod_{i} U_{i}^{n}$ is the product of spectral flow operators and $\chi_{\mathbf{h}, \mathbf{t}}(\tau, v)$ is the product of characters of minimal models. Hence, the transition amplitude calculates the number of open string states between the pair of D-branes weighted by $q^{L_{0}-\frac{c}{24}}$.

This space is given by the cohomology of the butterfly resolution (which is the product of the butterfly resolutions for individual minimal model).

The calculation of cohomology is given in two steps. At first step we calculate the cohomology with respect to the $\sum_{i} Q_{i}^{+}$-differential. It is well known that $\sum_{i} Q_{i}^{+}$-cohomology is generated by the set of $b c \beta \gamma$-system of fields

$$
\begin{array}{lc}
a_{i}(z)=\exp \left(X_{i}(z)\right), \quad a_{i}^{*}(z)=\left(\partial X_{i}^{*}-\psi_{i} \psi_{i}^{*}\right) \exp \left(-X_{i}(z)\right) \\
\alpha_{i}(z)=\psi_{i} \exp \left(X_{i}(z)\right), \quad \alpha_{i}^{*}(z)=\psi_{i}^{*} \exp \left(-X_{i}(z)\right)
\end{array}
$$

Their Fourier components commute with each other except the following

$$
\left[a^{*}[n] a[m]\right]=\delta_{n+m, 0}, \quad\left[\alpha^{*}[r], \alpha[s]\right]_{+}=\delta_{r+s, 0} .
$$

Hence, one can interpret these fields as a string version of the complex coordinates, the derivatives and differentials on the flat complex space $\mathbb{C}^{I}$ :

$$
\begin{aligned}
& a_{i}(z) \rightarrow \text { coordinates } a_{i} \text { on } \mathbb{C}^{I}, \quad a_{i}^{*}(z) \rightarrow \text { derivatives } \frac{\partial}{\partial a_{i}}, \\
& \alpha_{i} \rightarrow \text { differentials } d a_{i}, \quad \alpha_{i}^{*} \rightarrow \text { conjugates to } d a_{i} .
\end{aligned}
$$

In order for the correspondence be well defined we need to specify how the fields (3.3) change under a change of coordinates $a_{1}, \ldots, a_{I}$. For the new set of coordinates

$$
\tilde{a}_{i}=g_{i}\left(a_{1}, \ldots, a_{I}\right), \quad a_{i}=f_{i}\left(\tilde{a}_{1}, \ldots, \tilde{a}_{I}\right)
$$

this is determined in [25] by the formulas

$$
\begin{aligned}
& \tilde{a}_{i}(z)=g_{i}\left(a_{1}(z), \ldots, a_{I}(z)\right), \\
& \tilde{\alpha}_{i}(z)=g_{i, j}\left(a_{1}(z), \ldots, a_{I}(z)\right) \alpha_{j}(z), \tilde{\alpha}_{i}^{*}(z)=f_{i, j}\left(a_{1}(z), \ldots, a_{I}(z)\right) \alpha_{j}^{*}(z), \\
& \tilde{a}_{i}^{*}(z)=f_{i, j}\left(a_{1}(z), \ldots, a_{I}(z)\right) a_{j}^{*}(z)+f_{n, i, l}\left(a_{1}(z), \ldots, a_{I}(z)\right) g_{l, m}\left(a_{1}(z), \ldots, a_{I}(z)\right) \alpha_{n}^{*}(z) \alpha_{m}(z),
\end{aligned}
$$

where $g_{i, j}=\frac{\partial g_{i}}{\partial a_{j}}, f_{i, j}=\frac{\partial f_{i}}{\partial \tilde{a}_{j}}, f_{i, j, k}=\frac{\partial^{2} f_{i}}{\partial \tilde{a}_{j} \partial \tilde{a}_{k}}$ and the normal ordering of the operators is implied.

Then the $N=2$ Virasoro superalgebra takes the form

$$
\begin{aligned}
& G^{-}=\sum_{i} \alpha_{i} a_{i}^{*}, \quad G^{+}=\sum_{i}\left(1-\frac{1}{\mu_{i}}\right) \alpha_{i}^{*} \partial a_{i}-\frac{1}{\mu_{i}} a_{i} \partial \alpha_{i}^{*}, \\
& J=\sum_{i}\left(1-\frac{1}{\mu_{i}}\right) \alpha_{i}^{*} \alpha_{i}+\frac{1}{\mu_{i}} a_{i} a_{i}^{*}, \\
& T=\sum_{i} \frac{1}{2}\left(\left(1+\frac{1}{\mu_{i}}\right) \partial \alpha_{i}^{*} \alpha_{i}-\left(1-\frac{1}{\mu_{i}}\right) \alpha_{i}^{*} \partial \alpha_{i}\right)+\left(1-\frac{1}{2 \mu_{i}}\right) \partial a_{i} a_{i}^{*}-\frac{1}{2 \mu_{i}} a_{i} \partial a_{i}^{*} .
\end{aligned}
$$

We see that $G^{-}(z)$ is the string version of de Rham differential on $\mathbb{C}^{I}$ and due to (3.4) we can see the space of states generated by the fields (3.3) as a chiral de Rham complex on $\mathbb{C}^{I}$ introduced recently by Malikov, Shechtman and Vaintrob [25] for smooth manifolds. It is easy to see from (3.2) that GSO projection in the open string sector makes the orbifold of $\mathbb{C}^{I}$. Indeed, the operator $\exp (2 \imath \pi m J[0])$ in the summation over $m$ produces the projection of the space of states generated by $b c \beta \gamma$-fields on the subspace with integer $J[0]$-charge, while the operator $U^{n \mathbf{v}}$ in the summation over $n$ generates the twisted sectors for the $b c \beta \gamma$-fields. It allows to conclude 
that open string sector can be described in terms of chiral de Rham complex on the orbifold $\mathbb{C}^{I}$ GSO. This object has been introduced recently by Frenkel and Szczesny in [26].

Now we take the second step in the cohomology calculation, i.e. we calculate the cohomology with respect to $\sum_{i} Q_{i}^{-}$-differential. The screening charges $Q_{i}^{-}$can be expressed in terms of the fields (3.3) as

$$
Q_{i}^{-}=\oint d z \alpha_{i} a_{i}^{\mu_{i}-1}(z)
$$

Therefore $\sum_{i} Q_{i}^{-}$is Koszul differential associated with Landau-Ginzburg potential

$$
W\left(a_{i}\right)=\sum_{i} a_{i}^{\mu_{i}}
$$

It proves that $A$-type D-branes in Gepner model are fractional D-branes [27] on the LandauGinzburg orbifold. Notice that taking the $\sum_{i} Q_{i}^{-}$cohomology as the first step we get the same background geometry of $\mathbb{C}^{I} / G S O$ Landau-Ginzburg orbifold [11]. The similar analysis can be carried out also for the case of B-type branes [11].

Thus, a free field construction of D-branes allows to extract geometry from the purely algebraic Recknagel-Schomerus construction. It is interesting to develop in more details the D-brane geometry description in terms of chiral de Rham complex.

\section{Acknowledgements}

I thank the organizers of the Symmetry-2007 conference for their hospitality. This work was supported in part by grants RFBR-07-02-0799-a, SS6358.2006.2.

\section{References}

[1] Polchinski J., Dirichlet branes and Ramond-Ramond charges, Phys. Rev. Lett. 75 (1995), 4724-4727, hep-th/9510017.

Polchinski J., TASI lectures on D-branes, hep-th/9611050.

[2] Gepner D., Space-time supersymmetry in compactified string theory and superconformal models, Nuclear Phys. B 296 (1988), 757-778.

[3] Feigin B.L., Fuchs D.B., Invariant skew-symmetric differential operators on line and Verma modules over the Virasoro algebra, Funct. Anal. Appl. 16 (1982), 114-126.

Feigin B.L., Fuchs D.B., Representations of Virasoro algebra, in Seminar on Supermanifolds 5, Editors D. Leites, Reports of Dept. Math. Stockholm Univ., 1986.

[4] Malikov F.G., Feigin B.L., Fuchs D.B., Singular vectors in Verma modules over Kac-Moody algebras, Funktsional. Anal. i Prilozhen. 20 (1986), no. 2, 25-37, 96 (in Russian).

[5] Feigin B.L., Frenkel E., Representations of affine Kac-Moody algebras and bosonization, Physics and Mathematics of Strings, Vol. 271, World Sci. Publishing, Teaneck, 1990.

Feigin B.L., Frenkel E., Affine Kac-Moody algebras and semi-infinite flag manifolds, Comm. Math. Phys. 128 (1990), 161-189.

[6] Felder G., BRS Approach to minimal models, Nuclear Phys. B 317 (1989), 215-236.

Bernard D., Felder G., Fock representations and BRSt cohomology in SL(2) current algebra, Comm. Math. Phys. 127 (1990), 145-168.

[7] Dotsenko V.S., Fateev V.A., Conformal algebra and multipoint correlation functions in 2D statistical models, Nuclear Phys. B 240 (1984). 312-348.

Dotsenko V.S., Fateev V.A., Four point correlation functions and operator algebra in 2D conformal invariant field theory with central charge $c<1$, Nuclear Phys. B 251 (1985), 691-734.

[8] Dotsenko V.S., Solving the $S U(2)$ conformal field theory using the Wakimoto free field representation, Nuclear Phys. B 358 (1991), 547-570. 
[9] Parkhomenko S.E., BRST construction of D-branes in SU(2) WZW model, Nuclear Phys. B 617 (2001), 198-214, hep-th/0103142.

[10] Parkhomenko S.E., Free field construction of D-branes in $N=2$ minimal models, Nuclear Phys. B 671 (2003), 325-342, hep-th/0301070.

[11] Parkhomenko S.E., Free field approach to D-branes in Gepner models, Nuclear Phys. B 731 (2005), 360-388, hep-th/0412296.

[12] Parkhomenko S.E., Free field representation of permutation D-branes in Gepner models, J. Exp. Theor. Phys. 102 (2006), 902-919, hep-th/0512322.

[13] Ishikawa H., Watamura S., Free field realization of D-brane in group manifold, J. High Energy Phys. 2000 (2000), no. 8, 044, 23 pages, hep-th/0007141.

[14] Feigin B.L., Semikhatov A.M., Sirota V.A., Tipunin I.Yu., Resolutions and characters of irredicible representations of the $N=2$ superconformal algebra, Nuclear Phys. B 536 (1998), 617-656, hep-th/9805179.

[15] Schwimmer A., Seiberg N., Comments on the $N=2,3,4$ superconformal algebras in two dimensions, Phys. Lett. B 184 (1987), 191-196.

[16] Feigin B.L., Semikhatov A.M., Free field resolutions of the unitary $N=2$ super-Virasoro representations, hep-th/9810059.

[17] Cardy J.L., Boundary conditions, fusion rules and the Verlinde formula, Nuclear Phys. B 324 (1989), 581596.

[18] Sen A., Tachyon condensation on the brane antibrane system, J. High Energy Phys. 1998 (1998), no. 8, 012, 6 pages, hep-th/9805170.

[19] Gelfand S., Manin Yu., Methods of homological algebra, Springer-Verlag, Berlin, 1996.

[20] Okuda T., Takayanagi T., Ghost D-branes, J. High Energy Phys. 2006 (2006), no. 3, 062 , 39 pages, hep-th/0601024.

[21] Recknagel A., Schomerus V., D-branes in Gepner models, Nuclear Phys. B 531 (1998), 185-225, hep-th/9712186.

[22] Eguchi T., Ooguri H., Taormina A., Yang S.-K., Superconformal algebras and string compactification on manifolds with $S U(n)$ holonomy, Nuclear Phys. B 315 (1989) 193-221.

[23] Fuchs J., Schweigert C., Walcher J., Projections in string theory and boundary states for Gepner models, Nuclear Phys. B 588 (2000), 110-148, hep-th/0003298.

[24] Recknagel A., Permutation branes, J. High Energy Phys. 2003 (2003), no. 4, 041, 27 pages, hep-th/0208119.

[25] Malikov F., Schechtman V., Vaintrob A., Chiral de Rham complex, Comm. Math. Phys. 204 (1999), 439473, math.AG/9803041.

[26] Frenkel E., Schestney M., Chiral de Rham complex on orbifolds, math.AG/0307181.

[27] Diaconescu D., Gomis J., Fractional branes and boundary states in orbifold theories, J. High Energy Phys. 2000 (2000), no. 10, 001, 48 pages, hep-th/9906242. 